\title{
A SHORT THEORETICAL NOTE ON OPTICAL ABSORPTION OF AMORPHOUS GALLIUM ARSENIDE IN THE INFRARED RANGE
}

\author{
M. A. GRADO-CAFFARO and M. GRADO-CAFFARO
}

C/Julio Palacios 11, 9으 28029-Madrid, Spain

(Received 21 April 1997; In final form 22 July 1997)

\begin{abstract}
An approach to determine the optical absorption coefficient of amorphous GaAs in the far infrared range is developed. Results from this approach are compared with experiment and with other theoretical results. In our formulation, contributions corresponding to both dynamical and structural disorders are taken into consideration.
\end{abstract}

Keywords: Optical absorption coefficient; amorphous GaAs; far infrared range

\section{INTRODUCTION}

Amorphous III - V compounds are not sufficiently investigated from the point of view of optical properties. In particular, theoretical work is necessary to understand completely the mechanisms involved in the optical absorption of the above semiconductors; a deep knowledge on this subject is crucial for device applications. Certainly, the technological implications of the above problems become very relevant in the field of optical communications (fiber-optic systems, optoelectronics in general). In the following, we will present a formulation that contains two parts: a theoretical model and a part consisting of numerical computations so that a theoretical plot will be obtained. 


\section{FORMULATION}

The first-order spectrum for the infrared range in disordered GaAs can be expressed as follows [1-5]:

$$
\varepsilon_{2}(\omega) \approx k[g(\omega) * g(\omega)] \approx A \omega+k g(\omega)
$$

with

$$
A=a\left(\varepsilon_{0}-\varepsilon_{\infty}\right) \gamma_{c} \omega_{T O(c)}^{-1} \int_{0}^{R}(d r) \cdot \frac{\exp \left(-b r^{2}\right)}{\omega_{T O}(r)}
$$

where $k, a$ and $b$ are positive constants, $g(\omega)$ is the phonon density of states, the asterisk denotes convolution, TO stands for transversal optical mode, $r$ is distance, $(c)$ denotes crystalline state, $\gamma_{c}$ is the damping factor and the above definite integral is referred to an interval of length $R$. Eq. (1) is approximately valid for the far infrared range. By applying Laplace's transform, this equation converts into:

$$
[G(u)]^{2}-G(u)-\frac{A}{k u^{2}}=0
$$

where

$$
G(u)=\mathcal{L}[g(\omega)]=\int_{0}^{\infty} g(\omega) \exp (-u \omega) d \omega
$$

Then from Eq. (3), it is deduced:

$$
g(\omega)=\mathcal{L}^{-1}\left[\frac{1 \pm\left(1+\frac{4 A}{k u^{2}}\right)^{1 / 2}}{2}\right]
$$

Now, since $\alpha(\omega)=\omega \varepsilon_{2}(\omega)$ where $\alpha(\omega)$ is the absorption coefficient, we have:

$$
\alpha(\omega) \approx A \omega^{2}+k \omega \mathcal{L}^{-1}\left[\frac{1 \pm\left(1+\frac{4 A}{k u^{2}}\right)^{1 / 2}}{2}\right]
$$


By using numerical values of previous references and performing numerical computations consisting, in essence, of an iteration process combined with a refinement technique, from Eqs. (2), (5) we have obtained:

$$
\alpha(\omega) \approx 5.512 \times 10^{3} \exp \left[-0.0031686(\omega-250)^{2}\right]
$$

whose plot corresponds to the solid line of Figure 1. Formula (6) does not correspond exactly to expressions (2), (5) but it is a useful approximation. On the other hand, Figure 2 shows a theoretical result in Ref. [7].

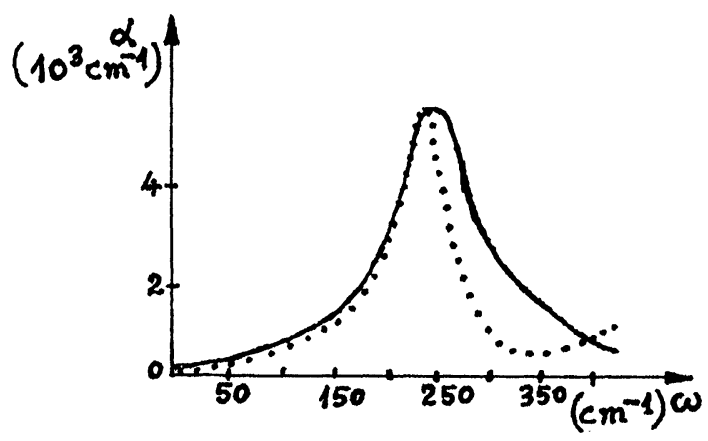

FIGURE 1 Plots of $\alpha(\omega)$ for a-GaAs in the far infrared range; the dotted line is experimental (after Ref. [6]) and the solid line is theoretical.

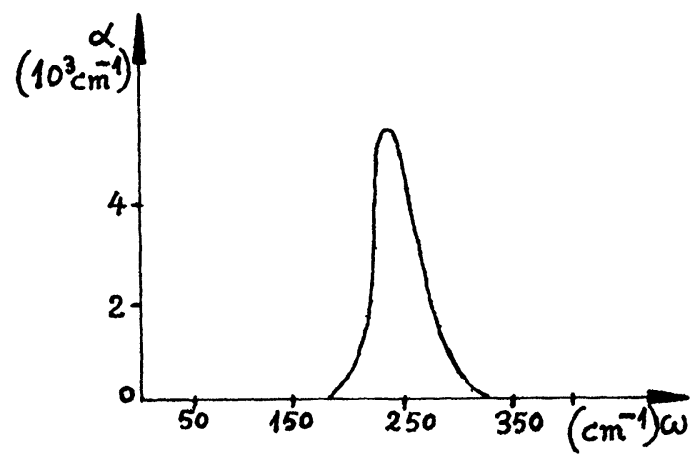

FIGURE 2 Theoretical plot of $\alpha(\omega)$ for a-GaAs in the far infrared range after Ref. [7]. 


\section{CONCLUDING REMARKS}

It is clear that the agreement of our results with experiment is much better than the plot of Figure 2 with the mentioned experimental curve. With respect to Figure 2, we can claim that for an approximate margin of $400 \mathrm{~cm}^{-1}$, only there is agreement between theory and experiment in the range $220 \mathrm{~cm}^{-1} \leq \omega \leq 320 \mathrm{~cm}^{-1}$ (approximately a quarter of the preceding entire range of $400 \mathrm{~cm}^{-1}$ ).

\section{References}

[1] Grado-Caffaro, M. A. and Grado-Caffaro, M. (1992). Phys. Lett., A 169, 399-401.

[2] Grado-Caffaro, M. A. and Grado-Caffaro, M. (1993). Mod. Phys. Lett., B 7, 1201-1207.

[3] Grado-Caffaro, M. A. and Grado-Caffaro, M. (1994). Act. Pass. Electronic Comp., 16, 109-112.

[4] Grado-Caffaro, M. A. and Grado-Caffaro, M. (1994). Mod. Phys. Lett., B 8, 169-172.

[5] Grado-Caffaro, M. A. and Grado-Caffaro, M. (1994). Mod. Phys. Lett., B8, 247-250.

[6] Prettl, W., Shevchik, N. J. and Cardona, M. (1973). Phys. Status Solidi (b), 59, 241.

[7] Mitra, S. S., Paul, D. K., Tsay, Y. F. and Bendow, B. (1974). AIP Conf. Proc. No. 20, 288. 

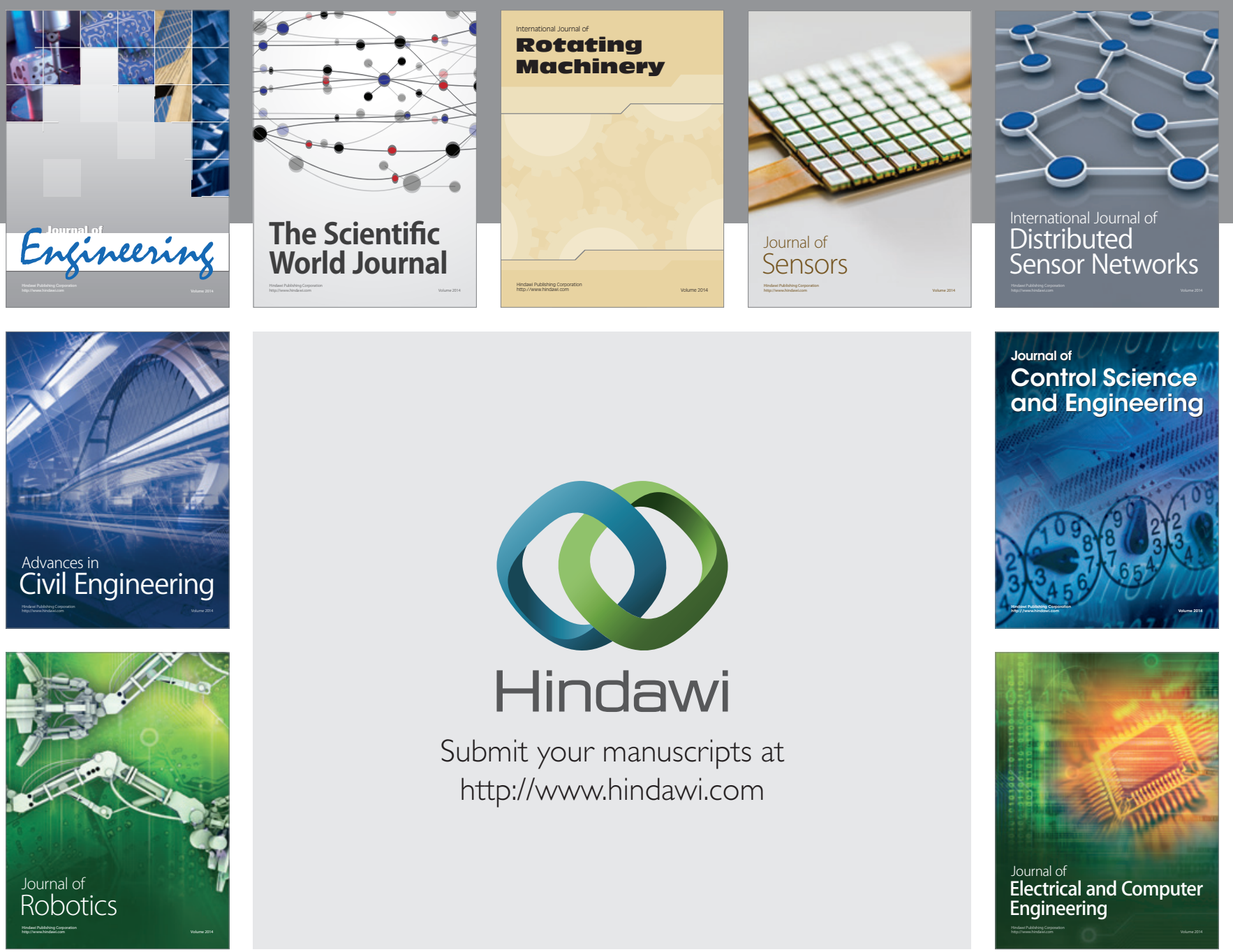

Submit your manuscripts at

http://www.hindawi.com
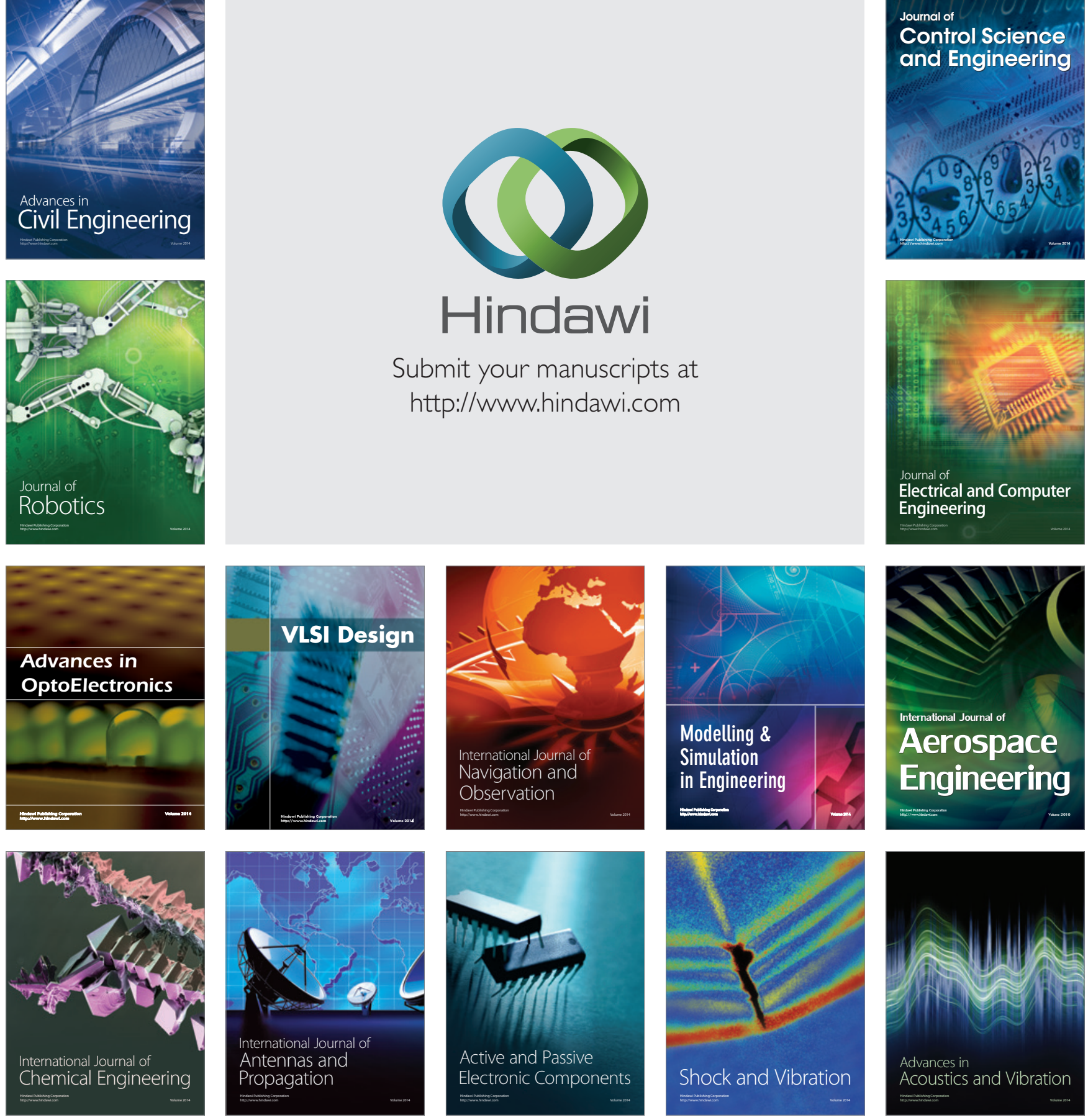